\title{
Plume deformation, mixing, and reaction kinetics: An analysis of interacting helical flows in three-dimensional porous media
}

\author{
Ye, Yu; Chiogna, Gabriele; Lu, Chunhui; Rolle, Massimo
}

Published in:

Physical Review E

Link to article, DOI:

10.1103/PhysRevE.102.013110

Publication date:

2020

Document Version

Publisher's PDF, also known as Version of record

Link back to DTU Orbit

Citation (APA):

Ye, Y., Chiogna, G., Lu, C., \& Rolle, M. (2020). Plume deformation, mixing, and reaction kinetics: An analysis of interacting helical flows in three-dimensional porous media. Physical Review E, 102(1), [013110].

https://doi.org/10.1103/PhysRevE.102.013110

\section{General rights}

Copyright and moral rights for the publications made accessible in the public portal are retained by the authors and/or other copyright owners and it is a condition of accessing publications that users recognise and abide by the legal requirements associated with these rights.

- Users may download and print one copy of any publication from the public portal for the purpose of private study or research.

- You may not further distribute the material or use it for any profit-making activity or commercial gain

- You may freely distribute the URL identifying the publication in the public portal 


\title{
Plume deformation, mixing, and reaction kinetics: An analysis of interacting helical flows in three-dimensional porous media
}

\author{
Yu Ye $\odot,{ }^{1,2}$ Gabriele Chiogna, ${ }^{3,4}$ Chunhui Lu, ${ }^{1,2}$ and Massimo Rolle $\odot^{5, *}$ \\ ${ }^{1}$ State Key Laboratory of Hydrology-Water Resources and Hydraulic Engineering, Hohai University, Nanjing 210098, China \\ ${ }^{2}$ Yangtze Institute for Conservation and Development, Hohai University, Nanjing 210098, China \\ ${ }^{3}$ Faculty of Civil, Geo, and Environmental Engineering, Technical University of Munich, Arcistraße 21, D-80333 Munich, Germany \\ ${ }^{4}$ Institute of Geography, University of Innsbruck, Innrain 52, 6020 Innsbruck, Austria \\ ${ }^{5}$ Department of Environmental Engineering, Technical University of Denmark, Miljøvej Building 115, DK-2800 Lyngby, Denmark
}

(Received 24 February 2020; accepted 6 July 2020; published 28 July 2020)

\begin{abstract}
Heterogeneity and macroscopic anisotropy of porous media play an important role for dilution and reaction enhancement of conservative and reactive plumes. In this study, we perform numerical simulations to investigate steady-state flow and transport in three-dimensional heterogeneous porous media. We consider two macroscopic anisotropic inclusions resulting in helical flows with twisting streamlines in a three-dimensional flow-through domain. The inclusions are obtained by alternating two layers of angled slices of coarse and fine porous media with different hydraulic conductivity. We investigate flow and transport scenarios considering different geometry and relative position of the two anisotropic inclusions yielding helical flow fields with different extent of interaction. We use metrics of stretching and folding to characterize the flow field and entropy-based metrics for the analysis of the conservative and reactive transport problems. The outcomes show that the two helices result in different patterns of twisting streamlines, which cause distinct deformation of the plumes. However, mixing and reaction enhancement could not be directly related to the extent of the flow field deformation: Configurations with strong deformation can result in only moderate mixing enhancement, whereas configurations with limited deformation of the flow field can lead to significant mixing of the solute plume. Finally, we explore the impact of different degradation rates on reactive transport and the role of reaction kinetics on the entropy balance for a reactant undergoing transport and mixing-controlled degradation in the twisting flow fields. The results show that strong mixing enhancement due to helical flow increases the importance of the reaction kinetics that becomes the rate-limiting process for solute reactive transport.
\end{abstract}

DOI: 10.1103/PhysRevE.102.013110

\section{INTRODUCTION}

The physics of mixing processes in fluids and their interactions with chemical and biological reactions are of paramount importance in both natural and engineered systems [1-9]. The investigation of these phenomena is a key focus of many scientific disciplines including fluid mechanics, chemical engineering, geochemistry, water treatment, and reservoir engineering, as well as subsurface and contaminant hydrology. Whereas in many applications entailing turbulent flows mixing is very effective, in porous media such as packed bed reactors and geologic formations mixing is determined by slow diffusive processes and often represents the rate-limiting step for chemical transformation and element cycling. A large number of studies investigated mixing processes in porous media with particular attention on the role of geologic heterogeneity in solute transport and mixing enhancement [10-20]. Mixing processes are crucial in many subsurface applications including contaminant transport and (bio)degradation, mineral precipitation and dissolution, viscous fingering, densitydriven convection, and groundwater-surface water interaction

*masro@env.dtu.dk
[21-28]. Most studies of mixing in heterogeneous porous media have been performed in two-dimensional (2D) setups including quasi two-dimensional flow-through experiments and detailed 2D numerical simulations [12,29-33], whereas fewer contributions have investigated mixing in fully three-dimensional (3D) systems [34-44]. Complex flows can develop in fully three-dimensional anisotropic porous media, entailing whirling and twisting streamlines [35,45-52]. Such 3D flow fields can cause significant deformation of dissolved solute plumes and ultimately result in considerable mixing enhancement. Recent contributions have provided experimental evidence of helical flow in macroscopically anisotropic porous media and quantified the impact of twisting flow on plume dilution in 3D flow-through setups $[53,54]$.

In this study we investigate streamline deformation, plume dilution, and reactive mixing in the presence of interacting helical flows. We perform numerical simulations in fully threedimensional setups with embedded macroscopic anisotropic inclusions causing helical flow and twisting streamlines. We consider scenarios with different location of the inclusions, orientation of the induced secondary motion, and different extent of interaction between the helical flows. We quantify streamline deformation, plume dilution, and its enhancement 


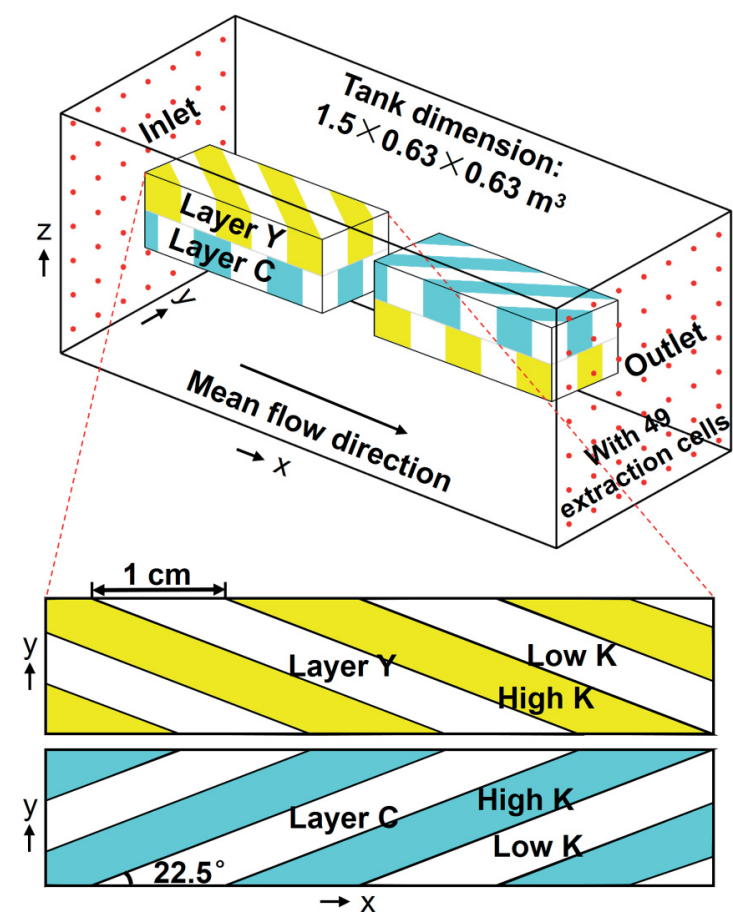

FIG. 1. Schematic of the 3D setup with two in-line macroscopic anisotropic inclusions. The bottom image shows a plan view of the two layers (Y: yellow and C: cyan) in one of the inclusions: colored slices represent zones of high hydraulic conductivity whereas white slices represent zones of low hydraulic conductivity.

due to the complex 3D flow fields. We also investigate reactive mixing and we explore the impact of reaction kinetics on the overall transport regime and on its physical mixing and/or (bio)chemical reaction limitation.

\section{PROBLEM STATEMENT}

In this work, we performed steady-state flow and conservative and reactive transport simulations in a fully threedimensional domain with dimensions $1.5 \mathrm{~m} \times 0.63 \mathrm{~m} \times 0.63 \mathrm{~m}$ (Fig. 1). Two macroscopic anisotropic structures $0.51 \mathrm{~m} \times$ $0.11 \mathrm{~m} \times 0.11 \mathrm{~m}$ (length $\times$ width $\times$ height) were embedded in the 3D domain. Forty-nine injection and extraction cells were used to simulate constant flow boundary conditions at the inlet and outlet surfaces of the flow-through system. No-flow was imposed at the other boundaries. The average seepage velocity used in the simulations was $3 \mathrm{~m} / \mathrm{d}$. For the study of conservative transport, a tracer was injected continuously through the central injection cell. In the reactive cases, we studied a bimolecular reaction of the form $A+B \rightarrow C$, representing a redox reaction between a dissolved organic compound and a dissolved electron acceptor. We consider both instantaneous and double Monod kinetics. In the case of instantaneous kinetics the overall transformation is mixing controlled, whereas in the case of double Monod formulation a range of mixing- and kinetically controlled conditions can be explored. Reactant $A$ was injected continuously through the central port at the inlet whereas the reactant $B$ was introduced parallel to $A$ within the ambient pore water solution.
Two anisotropic inclusions were inserted in an otherwise homogeneous matrix considering a total of 16 parallel and sequential configurations. The hydraulic conductivity of the matrix was $2.5 \times 10^{-3} \mathrm{~m} / \mathrm{s}$. Each inclusion was constructed with two layers alternating angled slices of high and low hydraulic conductivity representing coarse and fine porous media. Such configuration was inspired from herringbone cross stratification observed in subsurface sedimentary depositional environments [55,56] and also used in microfluidic devices [57]. Figure 1 shows the arrangement of the porous media in two different layers, i.e., layer Y and layer C. Colored slices represent the coarse medium with higher hydraulic conductivity (i.e., $3.0625 \times 10^{-2} \mathrm{~m} / \mathrm{s}$ ), whereas white slices represent the less permeable medium with the same hydraulic conductivity as the matrix. The grain diameter was computed applying the equation proposed by Hazen [58], i.e., $K=(C d)^{2}$, where $K\left[L T^{-1}\right]$ is the hydraulic conductivity, $d[L]$ is the grain diameter, and the proportionality constant $C=100 \mathrm{~m}^{-0.5} \mathrm{~s}^{-0.5}$ [58]. The grain diameters were 0.5 and $1.75 \mathrm{~mm}$ for the fine and the coarse medium, respectively, and such grain sizes were used in previous experimental studies [53,54]. Porosity was set to 0.4 for both fine and coarse materials as observed in previous experimental studies [54,59]. The angle between the slices and the longitudinal direction was set to $22.5^{\circ}$ and spacing between two slices in both layers was set to $1 \mathrm{~cm}$, since these parameters were found optimal for dilution and reactive mixing [43].

The position and the geometry of the two inclusions in the different configurations are shown in Figs. 2 and 3. Figure 2 shows the $y-z$ cross section of the eight configurations in which the heterogeneous anisotropic inclusions are deployed in parallel. Figure 3 shows $x-z$ cross sections for the other eight configurations in which the inclusions are embedded in series. In the eight scenarios illustrated in Fig. 2, the starting point of the inclusions was $0.2 \mathrm{~m}$ from the inlet along the longitudinal direction. In the $y$ and $z$ directions (i.e., transverse horizontal and vertical directions), four different relative positions between the two inclusions were selected. Simultaneously, two different geometries were constructed by exchanging the sequences of the layers in the inclusions, thus creating different combinations of helical flows. The notation $S$ represents the setups with inclusions having the same order of the layers (i.e., layer $\mathrm{Y}$ above layer $\mathrm{C}$ in both inclusions) and resulting in helical flow with same rotation, whereas the notation $\mathrm{O}$ denotes the setups with an opposite arrangement of layer $\mathrm{Y}$ and layer $\mathrm{C}$ between the two inclusions, thus resulting in helices with opposite rotation. In cases $\mathrm{S} 1$ and $\mathrm{O} 1$, the two inclusions were inserted at the center of the $y-z$ cross section and they were connected to each other on one side. The two inclusions were shifted in the $z$ direction and they reached a distance of one and two layers height for cases $\mathrm{S} 2$ and $\mathrm{O} 2$ and cases $\mathrm{S} 3$ and $\mathrm{O} 3$, respectively. In cases $\mathrm{S} 4$ and $\mathrm{O} 4$, a spacing of $0.05 \mathrm{~m}$ was present between the two inclusions in the $y$ direction.

Figure 3 shows the eight configurations with the inclusions in series, aligned with the central injection cell. The first inclusion started at $0.2 \mathrm{~m}$ in the $x$ direction for cases S5 and $\mathrm{O} 5$ and cases $\mathrm{S} 7$ and $\mathrm{O} 7$, and it was shifted $0.1 \mathrm{~m}$ ahead for the other cases. For cases S5, O5, S6, and O6 the two inclusions 


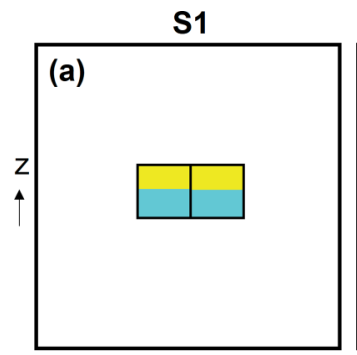

01

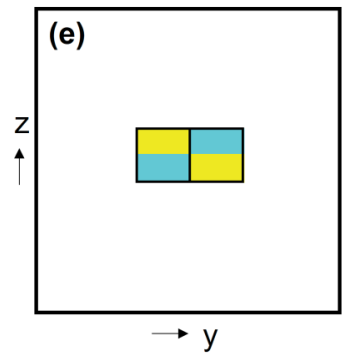

S2

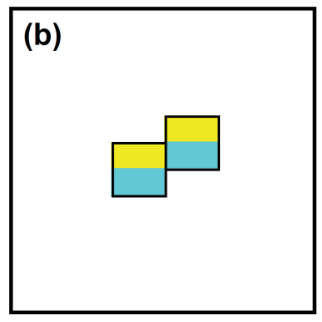

02

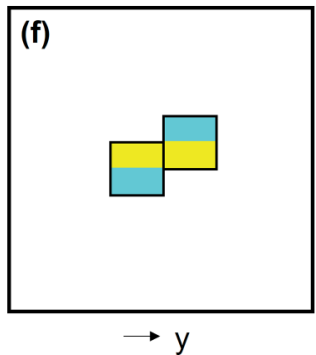

S3

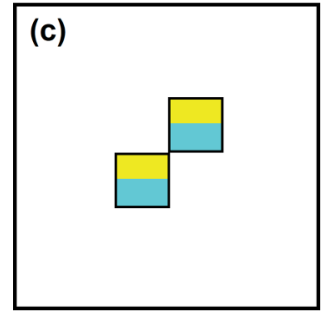

O3

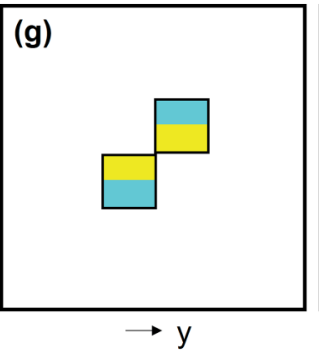

S4

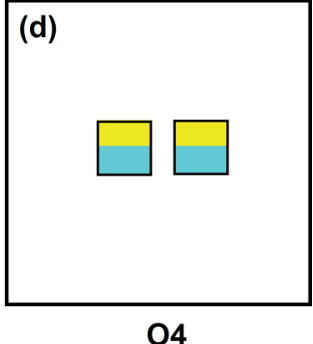

(h)

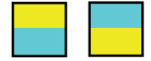

FIG. 2. Configurations with the two inclusions in parallel, shown as $y-z$ cross sections: yellow rectangles represent layers structured as layer $\mathrm{Y}$ and cyan rectangles represent layers structured as layer $\mathrm{C}$.

were connected, whereas in cases $\mathrm{S} 7, \mathrm{O} 7, \mathrm{~S} 8$, and $\mathrm{O} 8$ there law: was a spacing of $0.1 \mathrm{~m}$ between the two inclusions.

$$
\nabla \cdot(-\mathbf{K} \nabla \phi)=0
$$

\section{A. Governing equations}

Steady-state flow and transport equations were the governing equations describing the processes occurring in the three-dimensional flow-through system. The flow problem was solved by combining the continuity equation and Darcy's

S5

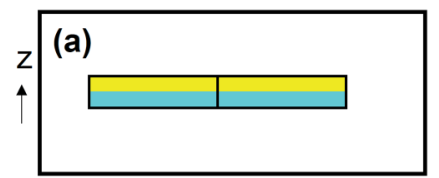

56

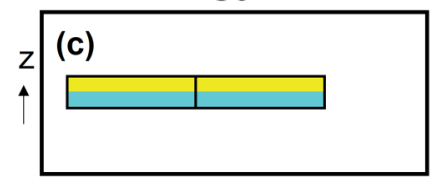

S7

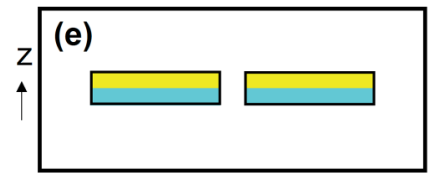

S8
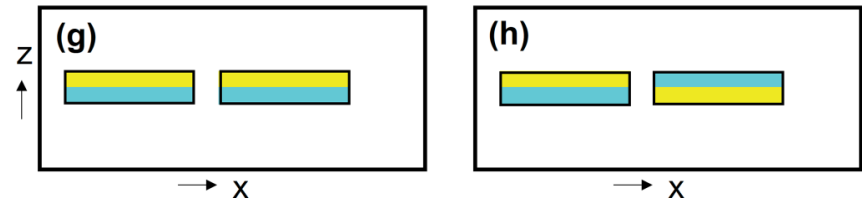

FIG. 3. Configurations with inclusions in series, shown as $x-z$ cross sections: Yellow rectangles represent layers structured as layer $\mathrm{Y}$ and cyan rectangles represent layers structured as layer $\mathrm{C}$.
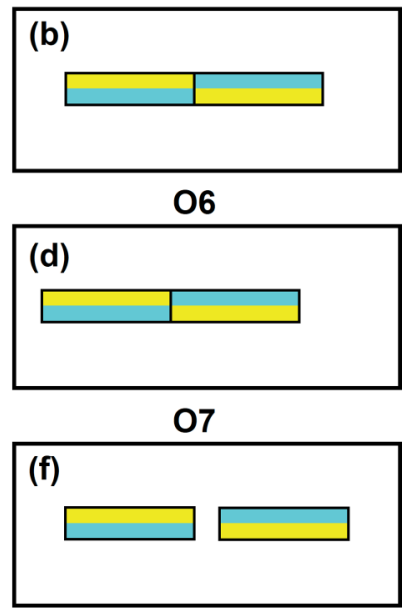

08 where $\mathbf{K}\left[L T^{-1}\right]$ is the hydraulic conductivity tensor and $\varphi[L]$ is the hydraulic head. Notice that although the porous medium is locally homogeneous and isotropic, at scales larger than the size of the layers it is characterized by an anisotropic heterogeneous hydraulic conductivity.

Transport was described by the advection-dispersionreaction equation:

$$
\mathbf{v} \cdot \nabla c_{i}-\nabla \cdot\left(\mathbf{D} \nabla c_{i}\right)=r_{i},
$$

where $\mathbf{v}\left[L T^{-1}\right]$ is the seepage velocity vector, $c_{i}\left[M L^{-3}\right]$ is the concentration of species $i, \mathbf{D}\left[L^{2} T^{-1}\right]$ is the hydrodynamic dispersion tensor, and $r_{i}\left[M L^{-3} T^{-1}\right]$ is the reaction rate of species $i$. The seepage velocity $\mathbf{v}$ equals the specific discharge $\mathbf{q}\left[L T^{-1}\right]$ divided by the porosity $\theta[-]$. In steady-state transport of a continuously emitted plume, the longitudinal dispersion term is of negligible importance compared to the transverse dispersion term that controls mixing of the plume with the surrounding groundwater [60]. The transverse dispersion coefficient $D_{t}\left[L^{2} T^{-1}\right]$ was described with a nonlinear compound-specific parametrization from previous experimental and pore-scale modeling investigation [31,61]:

$$
D_{t}=D_{p}+D_{a q}\left(\frac{\mathrm{Pe}^{2}}{\mathrm{Pe}+2+4 \delta^{2}}\right)^{\beta}
$$

where $D_{p}=\theta D_{a q}\left[L^{2} T^{-1}\right]$ is the pore diffusion coefficient, $D_{a q}\left[L^{2} T^{-1}\right]$ is the aqueous molecular diffusion coefficient, $P e=v d / D_{a q}[-]$ is the grain Péclet number, $v\left[L T^{-1}\right]$ is the magnitude of the seepage velocity, $\delta[-]$ is the ratio between the length of a pore channel and its hydraulic radius, and $\beta[-]$ is an empirical exponent that accounts for the degree of incomplete mixing within the pore channels. In the case of conservative transport, the reactive term equals zero. In the reactive transport simulations, we considered the 
mixing-controlled reaction between the species $A$ and $B$ that can react when they come in contact by mixing at the fringes of the injected plume of $A$. We considered both instantaneous and double Monod reaction kinetics. For instantaneous reactions, the reactants $A$ and $B$ cannot coexist and the reactant which is not in excess is always at 0 concentration (i.e., $c_{A} c_{B}=0$, where $c_{A}\left[M L^{-3}\right]$ and $c_{B}\left[M L^{-3}\right]$ are the concentrations of reactants $A$ and $B[62,63])$. The double Monod kinetics describes typical microbially mediated reactions in which the reaction rate is coupled to the biomass growth:

$$
r_{i}=-\frac{\mu_{\max }}{Y} \frac{c_{A}}{K_{A}+c_{A}} \frac{c_{B}}{K_{B}+c_{B}} c_{\text {bio }},
$$

where $\mu_{\max }\left[T^{-1}\right]$ is the maximum specific growth rate, $Y[-]$ is the specific yield coefficient, $K_{A}\left[M L^{-3}\right]$ and $K_{B}\left[M L^{-3}\right]$ are the half-saturation constants, and $c_{\text {bio }}\left[M L^{-3}\right]$ is the concentration of the microorganisms that mediate the redox reaction. Fast, intermediate, and slow double Monod kinetics were tested by varying $\mu_{\max }$ by two orders of magnitude. The biomass does not change with time under steady-state conditions, resulting from the dynamic balance between the growth and the linear decay terms:

$$
\mu_{\max } \frac{c_{A}}{K_{A}+c_{A}} \frac{c_{B}}{K_{B}+c_{B}} c_{\text {bio }}-k_{\text {dec }} c_{\text {bio }}=0,
$$

where $k_{\mathrm{dec}}\left[T^{-1}\right]$ is the biomass decay coefficient.

In the reactive transport scenarios, a virtual conservative compound $X[-]$ was used to simplify the solution of the transport problem [62]. $X$ is defined as the mixing ratio, which quantifies the volumetric ratio of the source-related water in the mixture with the ambient solution. A critical mixing ratio is defined at the fringe of the plume at which the concentrations of reactants $A$ and $B$ are in the stoichiometric ratio of the reaction:

$$
X_{\mathrm{crit}}=\frac{c_{B}^{\mathrm{amb}}}{c_{B}^{\mathrm{amb}}+c_{A}^{\mathrm{in}}} .
$$

At the locations where $X=X_{\text {crit }}$, both reactants have a zero concentration. The concentrations of the reactants and the products can be computed analytically from the mixing ratio [62]. Note that the application of this analytical approach requires an identical $D_{t}$ value for all transported species. For instantaneous reactions of $A+B \rightarrow C$, the concentration of reactant $A$ can be computed as

$$
c_{A}= \begin{cases}X c_{A}^{\mathrm{in}}-(1-X) c_{B}^{\mathrm{amb}} & X \geqslant X_{\text {crit }} \\ 0 & X \leqslant X_{\text {crit }}\end{cases}
$$

where $c_{A}^{\text {in }}\left[M L^{-3}\right]$ is the concentration of species $A$ at the inlet source and $c_{B}^{\mathrm{amb}}\left[M L^{-3}\right]$ is the concentration of species $B$ in the inlet ambient water. For double Monod reaction kinetics and considering the biomass at steady state, the concentration of reactant $A$ is computed as [62]

$$
c_{A}=X c_{A}^{\text {in }}+\frac{p_{1}+\sqrt{p_{1}^{2}-4 p_{0} p_{2}}}{2 p_{2}},
$$

TABLE I. Summary of flow, transport, and reaction parameters used in the model.

\begin{tabular}{lccc}
\hline \hline$K$, fine medium & $2.5 \times 10^{-3} \mathrm{~m} / \mathrm{s}$ & $\mu_{\max }$ & $0.5,5,50 / \mathrm{d}$ \\
$K$, coarse medium & $3.0625 \times 10^{-2} \mathrm{~m} / \mathrm{s}$ & $k_{\operatorname{dec}}{ }^{a}$ & $0.1 / \mathrm{d}$ \\
$\theta$ & 0.4 & $c_{A}^{\text {in }}$ & $0.07-0.7,1 \mathrm{mmol} / 1$ \\
$D_{a q}{ }^{\mathrm{b}}$ & $4.8 \times 10^{-10} \mathrm{~m}^{2} / \mathrm{s}$ & $c_{B}^{\text {amb }}$ & $0.7 \mathrm{mmol} / 1$ \\
$\delta$ in Eq. (3) ${ }^{\mathrm{c}}$ & 5.37 & $K_{A}{ }^{\mathrm{a}}$ & $8.33 \times 10^{-2} \mathrm{mmol} / 1$ \\
$\beta$ in Eq. (3) & 0.5 & $K_{B}{ }^{\mathrm{a}}$ & $3.13 \times 10^{-2} \mathrm{mmol} / 1$ \\
\hline \hline
\end{tabular}

${ }^{\mathrm{a}}$ From [62].

${ }^{\mathrm{b}}$ Value for the common tracer fluorescein $[65,66]$.

${ }^{\mathrm{c}}$ From [16].

where $p_{0}, p_{1}$, and $p_{2}$ are

$$
\begin{gathered}
p_{0}=X c_{A}^{\mathrm{in}}(1-X) c_{B}^{\mathrm{amb}}-\frac{k_{\mathrm{dec}}}{\mu_{\max }}\left(K_{A}+X c_{A}^{\mathrm{in}}\right)\left[K_{B}+(1-X) c_{B}^{\mathrm{amb}}\right] \\
p_{1}=\frac{k_{\mathrm{dec}}}{\mu_{\max }}\left[K_{A}+X c_{A}^{\mathrm{in}}+K_{B}+(1-X) c_{B}^{\mathrm{amb}}\right] \\
-\left[X c_{A}^{\mathrm{in}}+(1-X) c_{B}^{\mathrm{amb}}\right], \\
p_{2}=1-\frac{k_{\mathrm{dec}}}{\mu_{\max }} .
\end{gathered}
$$

Flow and transport in the three-dimensional setup were solved using the numerical approach proposed by Cirpka et al. [64] that can be summarized in the following main steps: (i) generation of heterogeneous anisotropic conductivity fields in a regular grid, (ii) solution of the flow problem with a finite element scheme, (iii) construction of streamline-oriented grids by particle tracking, (iv) placement of nodes along the streamlines in regularly spaced observation planes orthogonal to the mean direction of flow and construction of Voronoi polygons, and (v) simulation of steady-state solute transport on the irregular streamline-oriented grid with a finite volume approach. This approach has the advantage of minimizing numerical dispersion compared to conventional methods solving solute transport on fixed Cartesian grids [64]. The 3D flow-through system was discretized into 4762800 cells with cell size of $0.005 \mathrm{~m}$ in each direction. All the parameters used in the numerical simulations are listed in Table I.

\section{B. Metrics of streamline deformation and mixing}

The metrics of stretching and folding have been proposed to characterize kinematic properties of complex flow fields $[35,40,67,68]$. In this study, we calculate these two metrics to quantify the effects of the heterogeneous anisotropic inclusions and their different configurations on the helical flow fields induced in the three-dimensional setup.

Stretching (indicated as $A^{2}[-]$ ) quantifies the increase of the length of an interface and it is defined as the normalized $L_{2}$-norm squared of the affine deformation of the streamlines:

$$
A^{2}(\mathbf{x}, \Delta x)=\frac{1}{r_{0}^{2} N} \sum_{n=1}^{N}\left\|\mathbf{A d}^{n}(x)\right\|_{2}^{2},
$$


where $\mathbf{x}[L]$ is the vector of the spatial coordinates; $\Delta x[L]$ is the space interval along the $x$ direction; $N$ denotes the number of streamlines adopted at the inlet of the flow-through system which uniformly form a circle, and each of the $N$ streamlines has an initial distance of $r_{0}[L]$ from the central reference streamline at the inlet; $\mathbf{d}^{n}[L]$ is the distance of the central streamline from one of the surrounding streamlines forming the cluster; $n$ is the index of the $N$ streamlines; and $\mathbf{A}$ is the affine deformation matrix. Therefore, at the inlet, $\mathbf{d}^{n}(0)$ can be written as $\left[r_{0} \cos (2 \pi n / N), r_{0} \sin (2 \pi n / N)\right]$. Detailed explanation can be found in Chiogna et al. [35]. A is defined as

$$
\mathbf{A}=\mathbf{W} \mathbf{J}^{-1}-\mathbf{I},
$$

where $\mathbf{I}$ is the identity matrix, and $\mathbf{W}$ and $\mathbf{J}$ are defined as

$$
\begin{gathered}
\mathbf{W}=\sum_{n=1}^{N} \mathbf{d}^{n}(x) \otimes \mathbf{d}^{n}(x+\Delta x), \\
\mathbf{J}=\sum_{n=1}^{N} \mathbf{d}^{n}(x) \otimes \mathbf{d}^{n}(x) .
\end{gathered}
$$

The symbol $\otimes$ denotes the matrix product. Extensive discussion of the two metrics has been provided in previous studies $[35,67,69]$.

Folding (indicated as $D^{2}[-]$ ) represents the bending of the interface and it is defined as the normalized $L_{2}$-norm squared of the nonaffine part of the streamlines deformation:

$$
D^{2}(\mathbf{x}, \Delta x)=\frac{1}{r_{0}^{2} N} \sum_{n=1}^{N}\left\|\mathbf{d}^{n}(x+\Delta x)-(\mathbf{A}+\mathbf{I}) \mathbf{d}^{n}(x)\right\|_{2}^{2} .
$$

Entropy-based metrics were computed to quantify dilution and reactive mixing. In particular, the flux-related dilution index $E_{Q}\left[L^{3} T^{-1}\right]$, physically representing the volumetric flux transporting the solute mass flux at a given cross section along the main flow direction [10], was calculated for the different scenarios:

$$
E_{Q}(x)=\exp \left[-\int_{\Omega}\left[p_{Q}(\mathbf{x}) \ln p_{Q}(\mathbf{x})\right] q_{x}(\mathbf{x}) d \Omega\right],
$$

where $p_{Q}=\frac{c}{\int_{\Omega} c q_{x} d \Omega}\left[T L^{-3}\right]$ is the flux-weighted probability density function of solute mass, $c\left[M L^{-3}\right]$ is the concentration, $q_{x}\left[L T^{-1}\right]$ is the specific discharge in the $x$ direction, and $\Omega$ is the cross section perpendicular to the $x$ direction. For conservative transport and considering a continuously injected plume, $E_{Q}$ is a monotonically increasing function with respect to the travel distance and quantifies the increased dilution of the plume due to the transverse diffusive/dispersive fluxes. This metric was later applied to study reactive transport $[70,71]$ and its value measures the interplay between dilution and reactive mixing. In fact, the development of $E_{Q}$ is determined by the balance between a positive source term due to dilution and a negative sink term due to reaction in the governing transport equation of the entropy density [70]:

$$
\begin{gathered}
\mathbf{v} \cdot \nabla\left(-p_{Q} \ln p_{Q}\right)-\nabla \cdot\left[\mathbf{D} \nabla\left(-p_{Q} \ln p_{Q}\right)\right] \\
=\frac{1}{p_{Q}} \nabla p_{Q}{ }^{T} \mathbf{D} \nabla p_{Q}-\left(1+\ln p_{Q}\right) r^{*},
\end{gathered}
$$

where the reactive term $r^{*}$ is defined as

$$
r^{*}=\left(\frac{\partial p_{Q}}{\partial c}-\frac{1}{r} \frac{\partial^{2} p_{Q}}{\partial c^{2}} \nabla c^{T} \mathbf{D} \nabla c\right) r
$$

where $r\left[M L^{-3} T^{-1}\right]$ is the reaction rate. Therefore, an increase of the reactant's entropy corresponds to dilutiondominated transport whereas a decrease occurs when the reactive term dominates the entropy density balance. The spatial derivative of the natural logarithm of $E_{Q}$ [i.e., $d \ln \left(E_{Q}\right) / d x$ ] is an intuitive metric that facilitates the evaluation of the complex interplay between dilution and reactive mixing: When this metric is positive, dilution dominates, whereas when the derivative of $\ln \left(E_{Q}\right)$ is negative, the reactive process is predominant.

Another intuitive metric to characterize reactive transport, particularly in case of instantaneous reaction kinetics, is the plume length, $L[L]$. It is defined as the minimal distance from the source at which species $A$ is completely consumed within the entire cross section and quantifies the length of the continuously injected plume of species $A$. Simple analytical expressions have been proposed to compute the plume length in homogeneous isotropic porous media both in 2D [72] and in 3D [54]. In this study, we use the numerical results to quantify and compare the reactive plume length for all 16 configurations of interacting helical flows in the 3D domain.

\section{RESULTS AND DISCUSSION}

\section{A. Helical flow}

The complex heterogeneous velocity fields entailing interacting helical flows can be visualized in Fig. 4, which shows the transverse horizontal and vertical mean specific discharge components along the planes normal to the direction $x$. Note that the $y-z$ cross sections shown in Fig. 4 are not the complete cross sections of the 3D setup but represent a smaller portion of the cross section comprising the heterogeneous and anisotropic inclusions. The flow is focused in the high-permeability zones in the inclusions and secondary motions of the flow velocity are induced by the arrangement of the porous media in the two layers (Fig. 1). This causes helical flows in the three-dimensional flow-through setup. The helical direction is dependent on the geometry of the anisotropic inclusion: In the cases with layer Y above layer $\mathrm{C}$ a counterclockwise helix is generated, whereas the inclusion with layer $\mathrm{Y}$ below layer $\mathrm{C}$ results in a clockwise helix. Cases S5-S8 show that also in the configurations with the two inclusions in series, the flow field preserves a mean helical motion when the anisotropic inclusions induce rotation in the same direction. Conversely, cases $\mathrm{O} 5-\mathrm{O} 8$ show that if two consecutive inclusions lead to rotations in the opposite direction, then the mean flow field does not preserve a distinct helical motion.

Stretching and folding were computed according to Eqs. (12) and (16) considering the entire cross section of the flow-through system and Fig. 5 shows their variability as a function of the coordinate $x$ along the main flow direction. Four radii (i.e., $r_{0}=0.01,0.02,0.03$, and $0.04 \mathrm{~m}$ ) for initial particle location were considered to analyze the sensitivity of the results to this parameter. In all cases, 36 particles per 

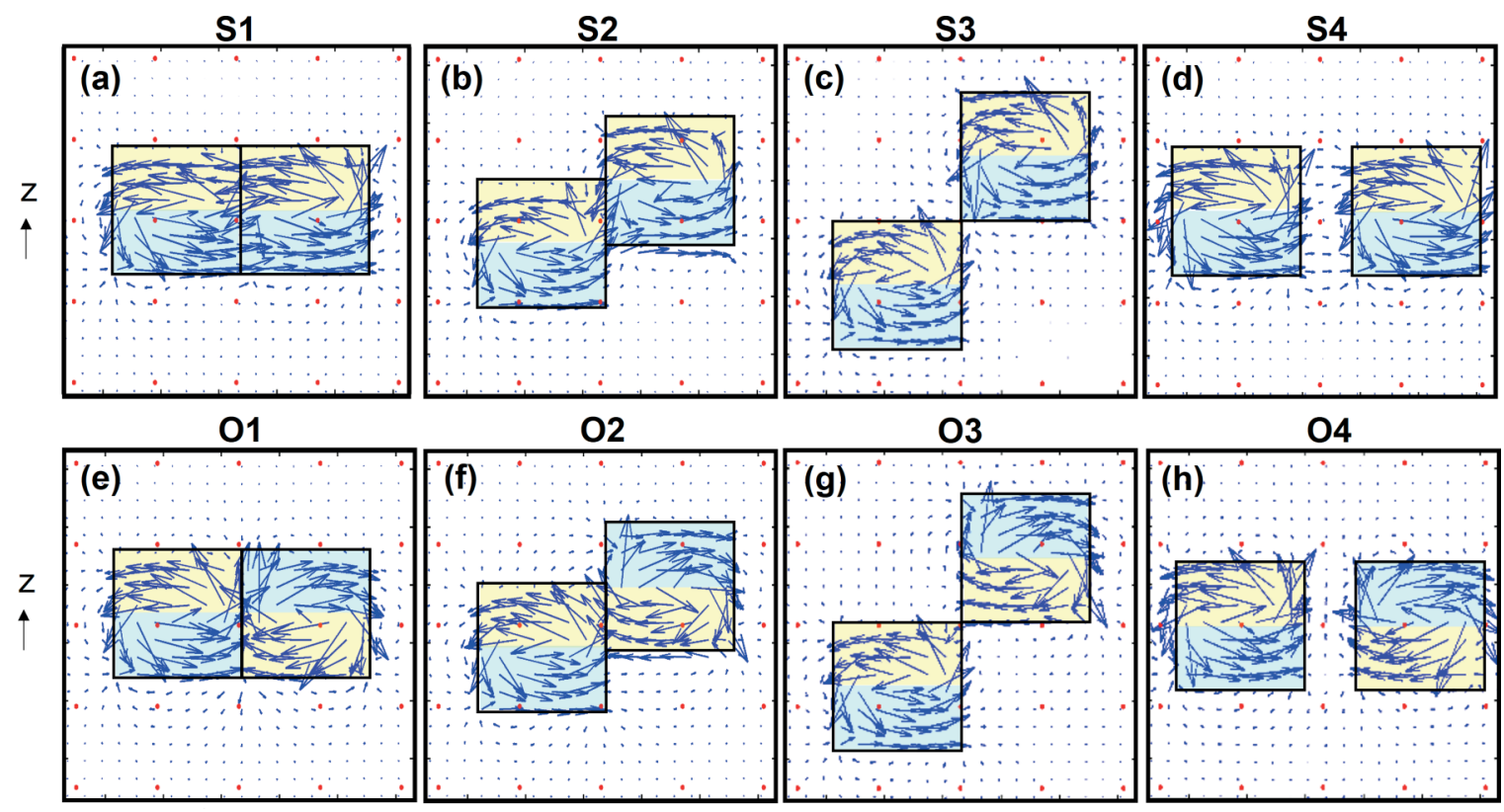

S5

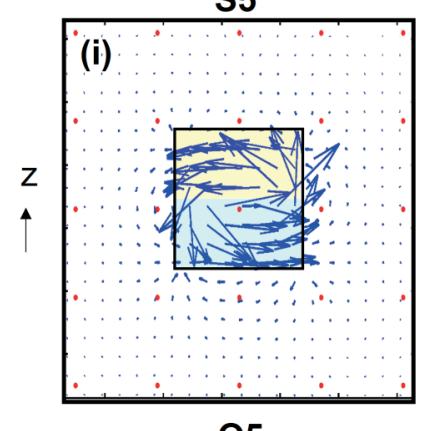

05

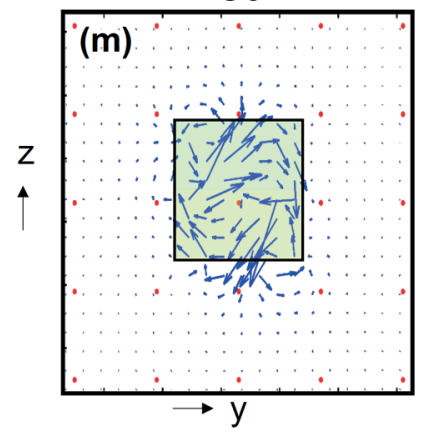

S6

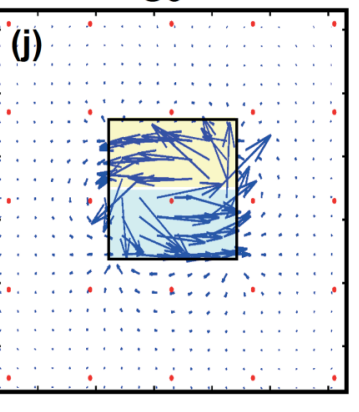

06

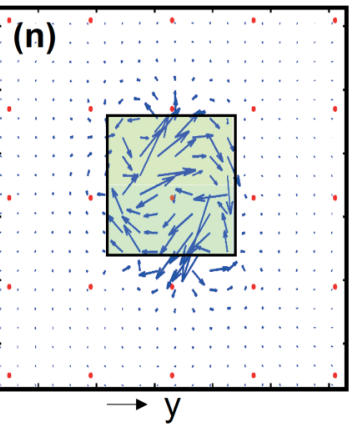

S7

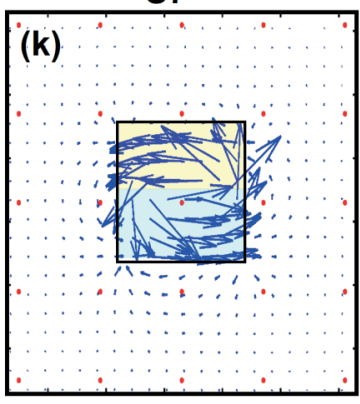

07

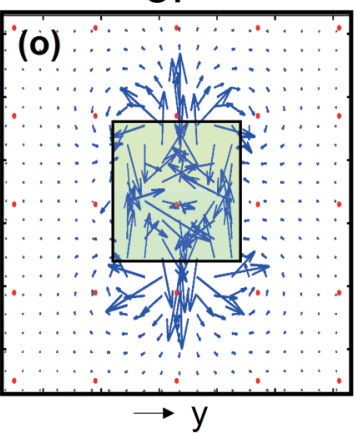

S8
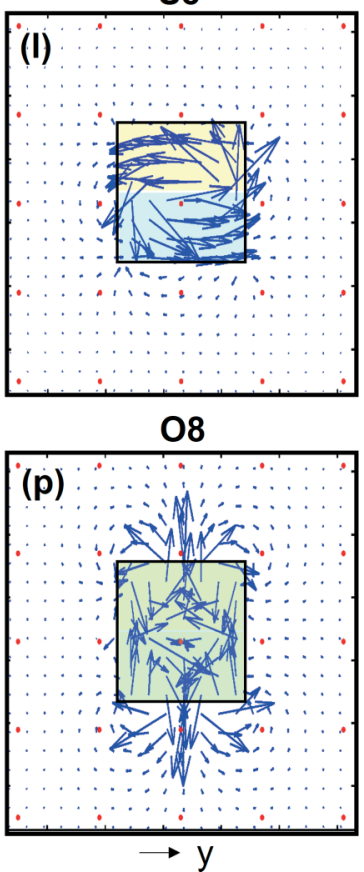

FIG. 4. Mean flow velocity at $y-z$ cross sections: The red dots represent the injection points, the blue arrows represent the specific discharge vectors, and the black boxes with colored shading indicate the position of the inclusions.

circle were used to obtain statistically significant results [67]. The helical flow caused by the structure and configuration of the inclusions leads to the variation of the values of the two kinematic quantities $\left\langle A^{2}\right\rangle$ and $\left\langle D^{2}\right\rangle$. On the contrary, the plots show that the values of $\left\langle A^{2}\right\rangle$ and $\left\langle D^{2}\right\rangle$ are the lowest and remain constant when the porous medium is homogeneous and the streamlines are straight. While the absolute values of $\left\langle A^{2}\right\rangle$ and $\left\langle D^{2}\right\rangle$ are dependent on the choice of the initial radius, the behavior of stretching and folding shows consistent patterns in the different cases. A similar behavior of stretching and folding was observed. This is due to the fact that the flow deformation occurs over a small portion of the domain occupied by the heterogeneous anisotropic inclusions. For the configuration $\mathrm{S} 1-\mathrm{O} 4$ (in parallel) only a length of $51 \mathrm{~cm}$ in the domain is affected by flow deformation, leading to a similar evolution of stretching and folding. In cases S5-O8 (in series) the differences are enhanced since the flow deformation occurs over a longer portion of the domain. Specifically, cases S5, S6, S7, and S8 display larger folding due to the same rotation of the streamlines in the flow field, whereas cases O5, O6, O7, and $\mathrm{O} 8$ show a decrease of stretching and folding in the second inclusion due to the opposite rotation of the helical flow. In such configurations (O5-O8) stretching and folding at the outlet reach the same initial values of the inlet. This shows that stretching and folding are reversible processes, contrary to mixing that is inherently irreversible. Such 

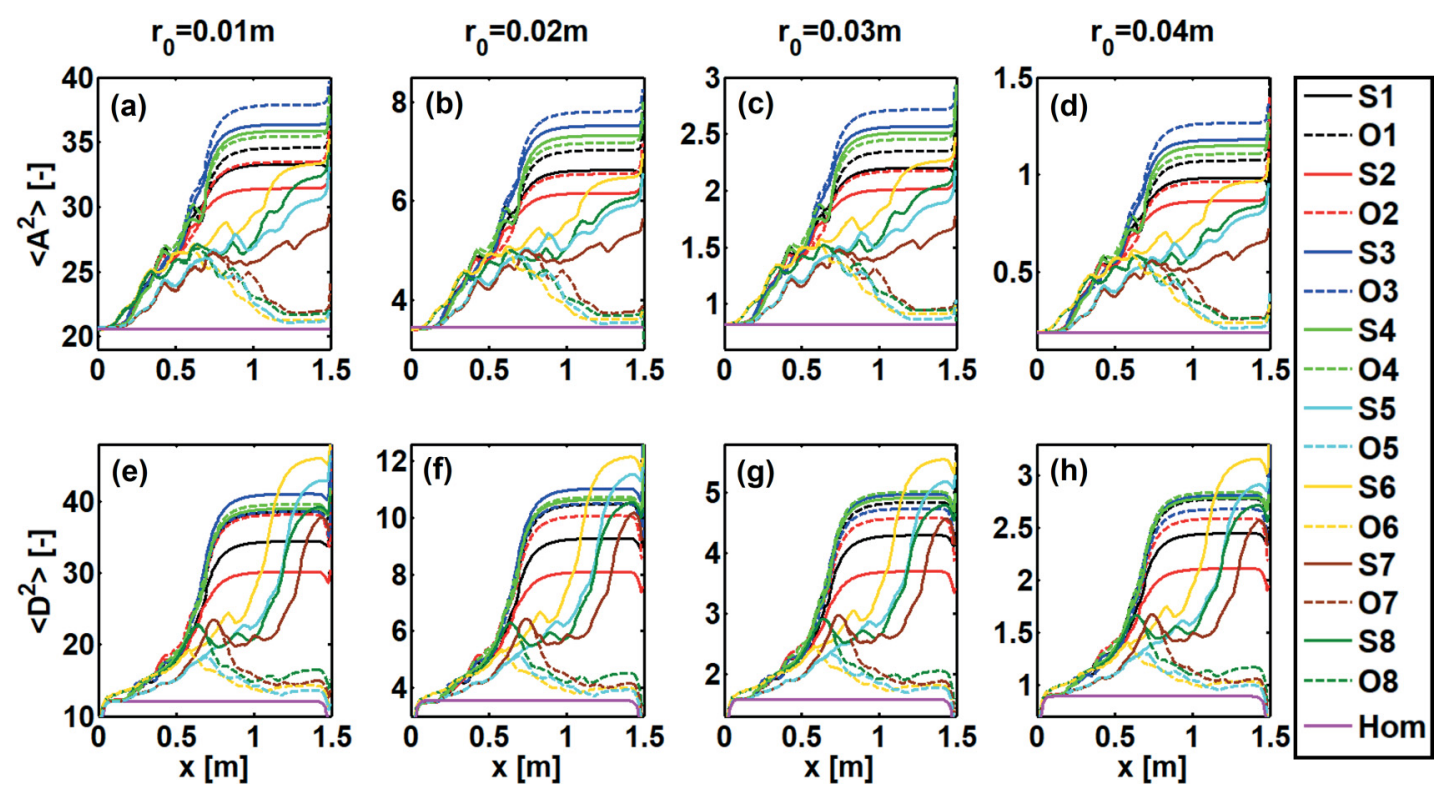

FIG. 5. Mean values of $A^{2}$ (a)-(d) and $D^{2}$ (e), (f) along the mean travel distance considering all 16 configurations and four different radii $\left(r_{0}\right)$ of initial location of the particles.

fundamental difference between the nonmonotonic behavior of these kinematic descriptors of the flow field compared to a monotonically increasing descriptor of mixing for conservative transport leads to a difficult interpretation of the kinematic results as predictors for mixing. Moreover, stretching and folding describe the properties of the entire flow field, while mixing processes concern the concentration field and are controlled by local diffusive/dispersive processes at the interface of the dissolved plume.

\section{B. Conservative transport}

The results of three-dimensional conservative transport for two selected configurations (case $\mathrm{O} 1$ and case S5) are shown in Fig. 6. Plumes injected at the center of the inlet cross section are significantly deformed by the complex flow field and the concentration decreases rapidly when the plume enters the heterogeneous anisotropic inclusions. The deformation of the plume occurs only within the inclusions, as predicted by the stretching and folding results. The deformed plumes are remarkably different in cases $\mathrm{O} 1$ and S5: The former does not display a peak in the middle of the cross section but has a ring
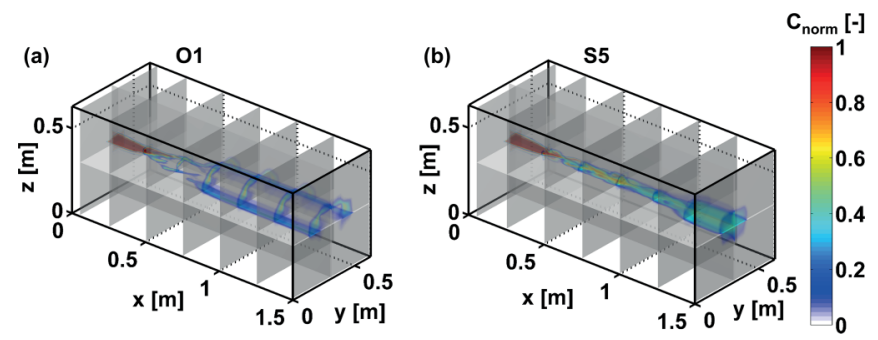

FIG. 6. Three-dimensional conservative plumes for case O1 (a) and case S5 (b). The colors represent the concentration of the conservative solute normalized by the initial value at the inlet. shape, whereas the latter maintains decreasing concentration from the core of the plume towards the outside.

The concentration distributions at the outlet cross section of the three-dimensional flow-through system for all cases are presented in Fig. 7. The panels in the first two rows [Figs. 7(a)-7(h)] illustrate the cases with the two anisotropic inclusions deployed in parallel, whereas the bottom panels refer to the inclusions in series [Figs. 7(i)-7(p)]. Cases S1S4 show that the plume splits into two separate parts with their own peak concentrations and the plume is approximately symmetric with respect to the center of the cross section, whereas cases $\mathrm{O} 1-\mathrm{O} 4$ show an asymmetric concentration distribution with respect to the center of the cross section. For the plumes in the configurations with sequential alignment of the anisotropic inclusions the plumes remain in the center of the domain and show the symmetric appearance of two (cases S5-S8) and four (cases O5-O8) wings, respectively.

Figure 8 shows the flux-related dilution index and its rate of increase along the travel distance for the 16 heterogeneous anisotropic setups and for a homogeneous porous medium. The trend of $E_{Q}$ is monotonically increasing in all setups and the anisotropic configurations show remarkable enhancement of plume dilution compared to the homogeneous case (i.e., no anisotropic inclusions in the 3D domain). The rate of increase of the flux-related dilution index shows that the enhancement of plume dilution happens in the portion of the domain where helical flow occurs (i.e., from the beginning of the first to the end of the second inclusion, $0.1<x<1.3 \mathrm{~m}$ ). Yet, the extent of dilution enhancement is dependent on the different helical flow patterns. For example, case $\mathrm{O} 1$ shows the highest dilution while case $\mathrm{O} 3$ presents the lowest dilution at the outlet of the flow-through system, resulting in a relative difference of $42.75 \%$. In the scenarios with the inclusions in series, dilution at the outlet is slightly higher in the cases with the two helices rotating in the same direction (i.e., cases S5-S8) in comparison to the corresponding scenarios with the two helices rotating in 


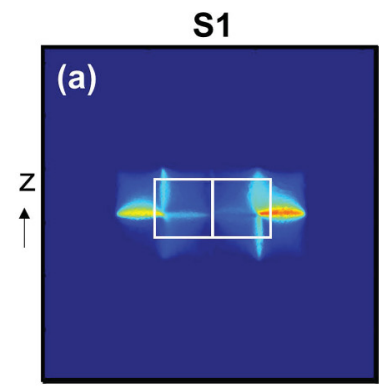

01

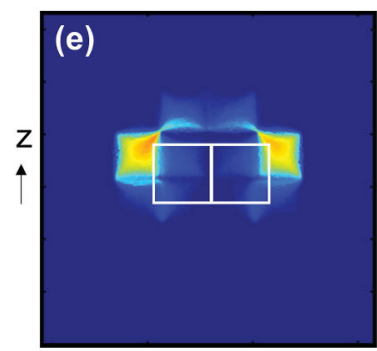

S5

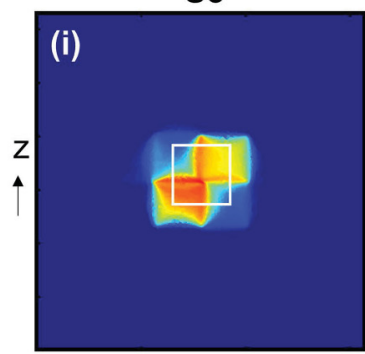

05

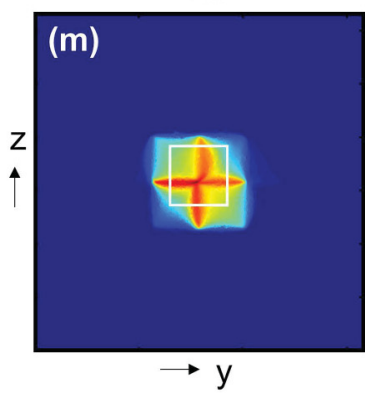

S2

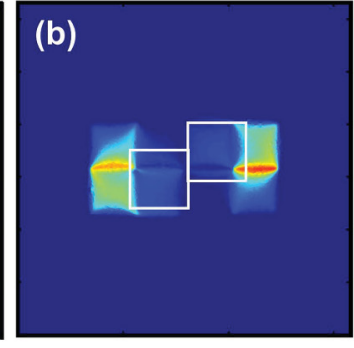

$\mathrm{O} 2$

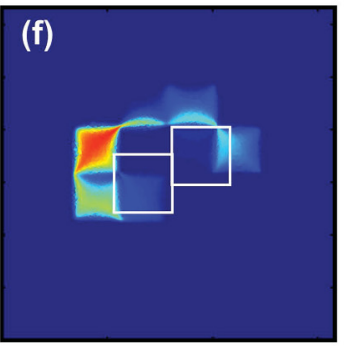

s6

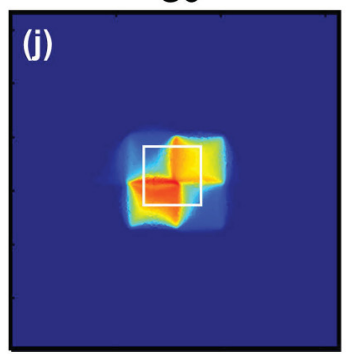

06

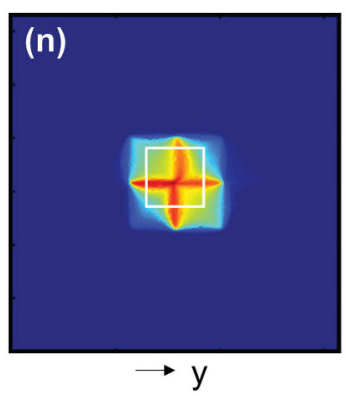

S3

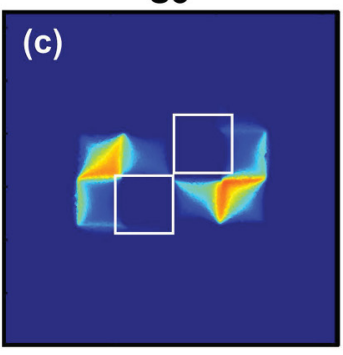

03

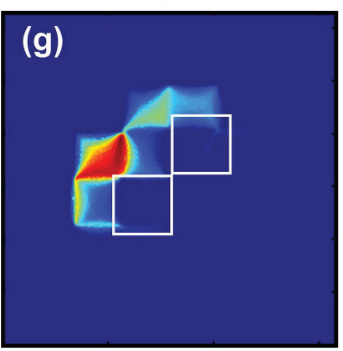

S7

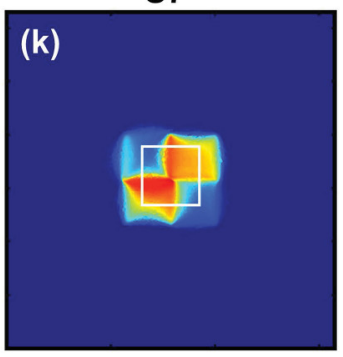

07

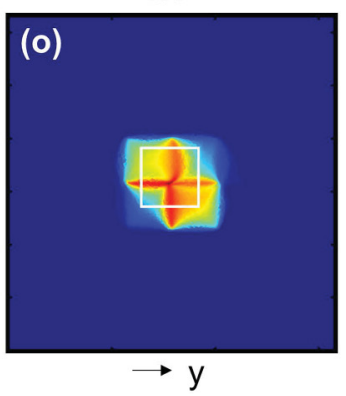

S4

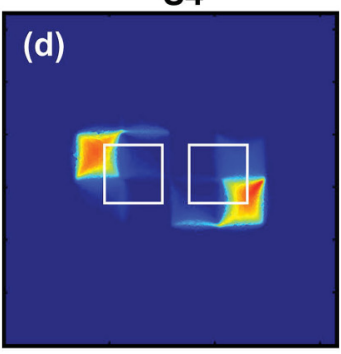

04

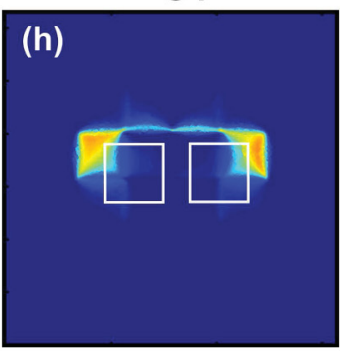

58

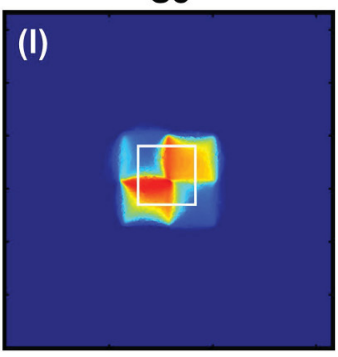

08

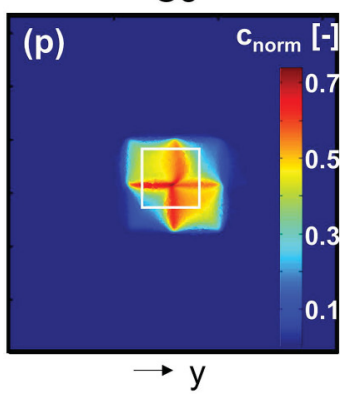

FIG. 7. Concentration distributions at the outlet cross section of the flow-through system for the 16 configurations. the colors represent the concentration of the conservative solute normalized by the value at the inlet, and the white boxes indicate the position of the inclusions.

the opposite direction (i.e., cases O5-08). Furthermore, the maximum dilution achieved in the setups with two sequential anisotropic inclusions is $4.80 \times 10^{-7} \mathrm{~m}^{3} / \mathrm{s}$, and it is smaller compared to the maximum dilution in the setups with the two anisotropic inclusions deployed in parallel (i.e., maximum $E_{Q}$ equals $5.97 \times 10^{-7} \mathrm{~m}^{3} / \mathrm{s}$ ). Dilution enhancement generally occurs in correspondence of changes in streamline stretching and folding. However, the extent of dilution enhancement cannot be directly predicted from the magnitude of these kinematic quantities. In fact, such quantities are descriptors for the entire flow field, while the plume does not occupy the entire cross section of the system. Furthermore, as discussed above, these kinematic metrics can have a reversible trend whereas dilution is monotonically increasing. For instance, in case $\mathrm{S} 2$ the deformation of the streamlines is the least significant among all the heterogeneous scenarios, yet the plume dilution is rather strong. Conversely, case O3 shows remarkable streamline deformation but the weakest dilution. Such a phenomenon is even more prominent in the cases with two opposite sequential helices (i.e., cases O5-08) in which the overall streamline deformation is minor but dilution enhancement is remarkable.

We can observe in particular that the direction of the rotation of the helical flow field has a minor impact on dilution enhancement if only the fringe of the plume is affected by the secondary motion (cases 4-8; see also Fig. 4). On the contrary, if plume deformation also impacts the central core of the plume, the asymmetry in the rotation generated by the heterogeneous anisotropic inclusions has important consequences on dilution enhancement (cases 1-3; see also Fig. 4). 


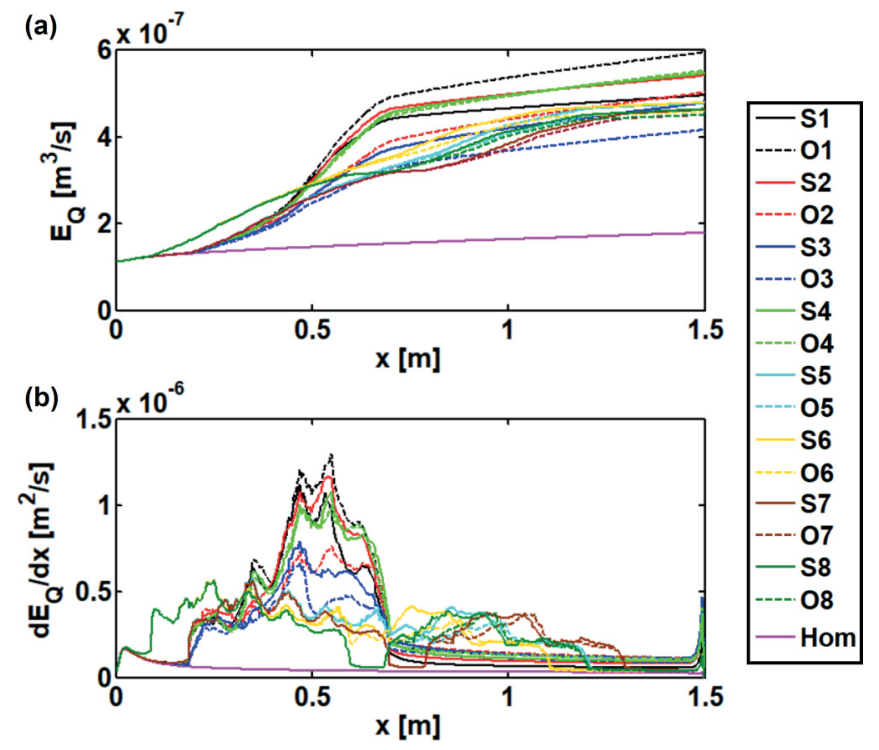

FIG. 8. Flux-related dilution index (a) and its rate of increase (b) along the longitudinal mean flow direction.

\section{Reactive transport \\ 1. Plume length}

Figure 9 shows the computed plume length at different critical mixing ratios considering the instantaneous reaction between the injected plume $A$ and reactant $B$ present in the injected ambient groundwater. In all heterogeneous anisotropic cases, the plumes can be shorter than the computational domain, whereas in the homogeneous case the plume would be always longer at the selected $X_{\text {crit }}$ range. An increase of critical mixing ratio represents a decrease of the concentration of the injected reactant $A$ [Eq. (6)] and results in a decrease of the plume length of reactant $A$. We can observe two distinct behaviors between cases $1-4$ and cases $5-8$ both in $S$ and $\mathrm{O}$ configurations. The first group shows a sudden decrease in the plume length, whereas the second one is characterized by a smoother decrease. The turning point of the first group corresponds to the plume length of $L=0.7 \mathrm{~m}$, which is the

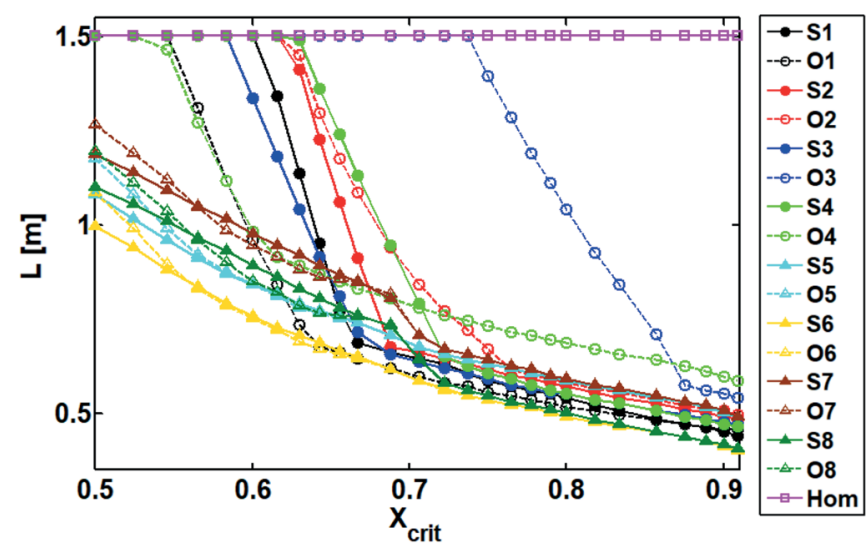

FIG. 9. Plume length computed for all 16 heterogeneous anisotropic configurations and for the homogeneous case considering various critical mixing ratios and instantaneous reaction kinetics.
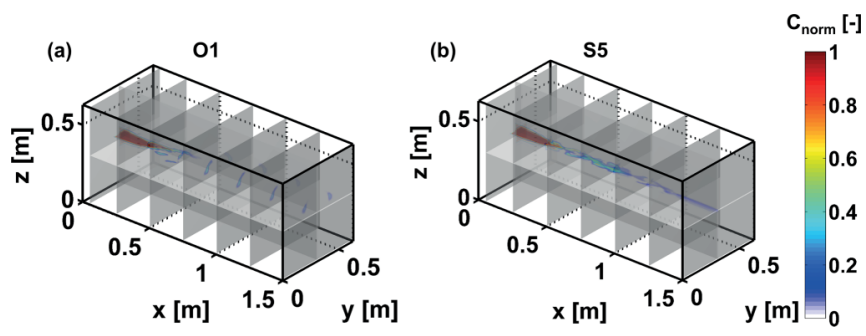

FIG. 10. Three-dimensional reactive plumes of species $A$ for case $\mathrm{O} 1$ (a) and case S5 (b), considering the intermediate double Monod kinetics. The colors represent the concentration of species $A$ normalized by the value at the inlet.

position where the inclusions ends in cases $1-4$. When the critical mixing ratio is small (i.e., the initial concentration of reactant $A$ is large compared to reactant $B$ ) and the plume is not yet consumed at the end of the inclusions, the mixing is limited in the homogeneous porous medium in the downgradient portion of the domain. In these conditions, a small increase of the initial concentration of reactant $A$ (i.e., a small decrease of the critical mixing ratio) leads to a steep increase of the plume length.

\section{Interplay between mixing and reaction kinetics}

The interplay between mixing and reaction kinetics was investigated by considering instantaneous, as well as fast, intermediate, and slow double Monod rates in the reactive transport simulations. Case $\mathrm{O} 1$ and case S5 were selected as representative cases with the two anisotropic inclusions in parallel and in series, respectively. These two cases were selected due to their high plume dilution. Figure 10 shows the three-dimensional reactive plumes for the two selected cases considering the intermediate double Monod kinetics. The reactant $A$ is degraded significantly within the anisotropic inclusions, indicating a reactive mixing enhancement by the induced helical flow.

The entropy development of reactant $A$ along the mean travel distance was computed for case O1 and case S5. Figure 11 shows the flux-related dilution index and the spatial derivative of its natural logarithm for reactant $A$ considering different reaction kinetics. The entropy is also plotted for the conservative transport case and, besides the two selected heterogeneous and anisotropic configurations, the results are also shown for a homogeneous porous medium. The entropy development differs among the scenarios with different reaction kinetics. In the instantaneous reactive cases, the entropy is mainly decreasing due to the instantaneous consumption of reactant $A$. The degradation of reactant $A$ is boosted within the inclusions, indicating a reactive mixing enhancement by the induced helical flows. When considering the slow double Monod kinetics the pattern of the reactant's flux-related dilution index is similar to the conservative case and maintains the monotonically increasing behavior showing that dilution dominates the entropy density balance. The scenarios with intermediate and, particularly, the one with fast Monod kinetics appear to approach the dilution trend observed in the cases with instantaneous reaction. The plots showing the spatial derivative of the natural logarithm of $E_{Q}(A)$ clearly illustrate 

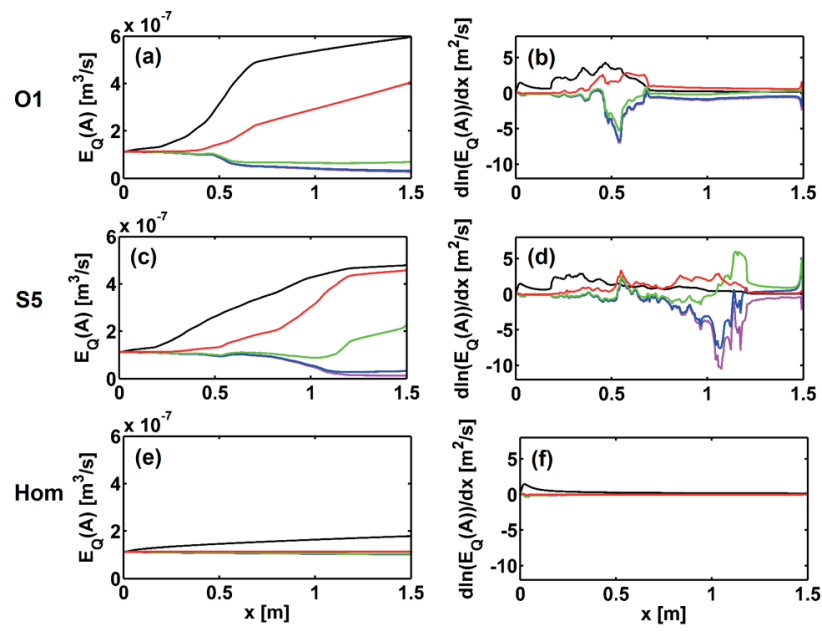

- Conservative - Instantaneous - Monod Fast - Monod Intermediate - Monod Slow

FIG. 11. Flux-related dilution index of reactant $A$ and spatial derivative of its natural logarithm for case O1 (a), (b), case S5 (c), (d), and homogeneous (e), (f) porous media setups, considering different reaction kinetics: conservative (black), instantaneous (magenta), double Monod fast (blue), double Monod intermediate (green), and double Monod slow (red) reaction kinetics.

the interplay between dilution and reactive processes in the anisotropic setups. The rate of increase of the flux-related dilution index is positive for the conservative case and is enhanced in the presence of the anisotropic inclusions. Similarly, the reactive cases considering a slow kinetic reaction show positive values of $d \ln \left[E_{Q}(A)\right] / d t$. When the reaction rates become faster the reactive term is more important and clearly becomes the main contribution in the entropy density balance for the cases of fast double Monod and instantaneous kinetics.

It is also interesting to compare the heterogeneous anisotropic cases with transport in the equivalent homogeneous domain. While the difference of entropy development among the four investigated reaction kinetics is remarkable in the heterogeneous setups only minor differences can be appreciated in the homogeneous porous medium [Figs. 11(e) and 11(f)]. In fact, mixing is weak in the homogeneous domain and changes in the reaction kinetics do not significantly impact the overall degradation, which remains mixing limited independently of the reaction rate. Conversely, in the heterogeneous anisotropic setups mixing is significantly enhanced by the helical flow that favors contact between the two reactants. The results show that, with sufficient mixing enhancement, the reaction kinetics is the limiting factor to the overall degradation and the trend of the reactive plume dilution becomes very sensitive to the rates of the biochemical reaction.

\section{SUMMARY AND CONCLUSIONS}

In this work, we numerically investigated the effect of interacting helical flows on solute transport and mixing in three-dimensional heterogeneous anisotropic porous media. The computed metrics of stretching and folding indicate deformed and twisted streamlines in the different configurations analyzed. The deformation depends on the position of the anisotropic inclusions and on the resulting helical flow patterns. The flux-related dilution index of conservative and reactive dissolved species and its derivative were computed to quantify dilution and reactive mixing for the conservative and reactive transport problems. Significant enhancement of dilution and reactive mixing results from the twisting streamlines which can deform the material surface of the plume and increase the transverse diffusive/dispersive fluxes. Our work shows that it is in general not possible to establish a direct correlation between stretching and folding and plume dilution and mixing. This can be due to the fact that the plume can occupy only a small portion of the flow field and/or the helical motion can differently affect the fringe and the core of the dissolved plume. Such findings also highlight the relevance of local concentration gradients and diffusive/dispersive effects specifically affecting the mass transfer and reactions at the interface between the dissolved plume and the surrounding pore water. Finally, we analyzed the interplay of physical mixing processes and biochemical reaction kinetics in the complex 3D setups. The outcomes show that once mixing is sufficiently enhanced the impact of different reaction rates becomes more important. This finding is interesting since it shows that the typically mixing-controlled regime of transport in porous media, originating from the creeping flow and the slow diffusive mass transfer, can be shifted towards being kinetically controlled. In fact, the considerable mixing enhancement by 3D helical flow causes the heterogeneous anisotropic setups to deviate from the poorly mixed behavior of common transport problems in porous media. In this situation the systems approach conditions common in other environmental flows, which are not dominated by viscous forces, where mixing is more effective and reaction kinetics are the overall rate-limiting steps.

\section{ACKNOWLEDGMENTS}

This study was supported by the National Natural Science Foundation of China (Grant No. 51709085) and the Fundamental Research Funds for the Central Universities (Grant No. B200202158). C.L. acknowledges the financial support of the National Key Research Project of China (Grant No. 2018YFC0407200) and the National Natural Science Foundation of China (Grants No. 51679067 and No. 51879088). M.R. acknowledges the support of the Independent Research Fund Denmark (GIGA Project No. DFF 7017-00130B) and of the Sino-Danish Center for Education and Research (SDC). G.C. acknowledges the support of the Stiftungsfonds für Umweltökonomie und Nachhaltigkeit GmbH (SUN) and the German Research Foundation (DFG, Project Hydromix CH 981/4-1). Constructive comments of two anonymous reviewers helped improve the quality of the manuscript.
[1] Z. Koza and H. Taitelbaum, Phys. Rev. E 56, 6387 (1997).
[2] H. A. Stone, A. D. Stroock, and A. Ajdari, Annu. Rev. Fluid Mech. 36, 381 (2004). 
[3] E. Villermaux, A. D. Stroock, and H. A. Stone, Phys. Rev. E 77, 015301(R) (2008).

[4] J. B. Weiss and A. Provenzale, Transport and Mixing in Geophysical Flows (Springer, Berlin, Heidelberg, 2008).

[5] Y. Deng, Z. Liu, P. Zhang, Y. Liu, Q. Gao, and Y. Wu, Biomed. Microdevices 14, 929 (2012).

[6] M. A. Budroni, L. A. Riolfo, L. Lemaigre, F. Rosso, M. Rustici, and A. De Wit, J. Phys. Chem. Lett. 5, 875 (2014).

[7] F. Haudin, M. Callewaert, W. De Malsche, and A. De Wit, Phys. Rev. Fluids 1, 074001 (2016).

[8] M. Rolle and T. Le Borgne, Rev. Mineral. Geochem. 85, 111 (2019).

[9] A. J. Valocchi, D. Bolster, and C. J. Werth, Transp. Porous Media 130, 157 (2019).

[10] M. Rolle, C. Eberhardt, G. Chiogna, O. A. Cirpka, and P. Grathwohl, J. Contam. Hydrol. 110, 130 (2009).

[11] M. Dentz and D. Bolster, Phys. Rev. Lett. 105, 244301 (2010).

[12] F. P. J. de Barros, M. Dentz, J. Koch, and W. Nowak, Geophys. Res. Lett. 39, L08404 (2012).

[13] B. Bijeljic, A. Raeini, P. Mostaghimi, and M. J. Blunt, Phys. Rev. E 87, 013011 (2013).

[14] P. de Anna, T. Le Borgne, M. Dentz, A. M. Tartakovsky, D. Bolster, and P. Davy, Phys. Rev. Lett. 110, 184502 (2013).

[15] T. Le Borgne, M. Dentz, and E. Villermaux, Phys. Rev. Lett. 110, 204501 (2013).

[16] Y. Ye, G. Chiogna, O. A. Cirpka, P. Grathwohl, and M. Rolle, Water Resour. Res. 51, 5582 (2015).

[17] M. Di Dato, F. P. J. de Barros, A. Fiori, and A. Bellin, Water Resour. Res. 52, 6818 (2016).

[18] M. R. Soltanian, M. A. Amooie, Z. Dai, D. Cole, and J. Moortgat, Sci. Rep. 6, 35921 (2016).

[19] M. Boon, B. Bijeljic, and S. Krevor, Water Resour. Res. 53, 4624 (2017).

[20] R. Turuban, D. R. Lester, J. Heyman, T. Le Borgne, and Y. Méheust, J. Fluid Mech. 871, 562 (2019).

[21] M. De Simoni, J. Carrera, X. Sánchez-Vila, and A. Guadagnini, Water Resour. Res. 41, W11410 (2005).

[22] R. D. Bauer, M. Rolle, S. Bauer, C. Eberhardt, P. Grathwohl, O. Kolditz, R. U. Meckenstock, and C. Griebler, J. Contam. Hydrol. 105, 56 (2009).

[23] B. Jha, L. Cueto-Felgueroso, and R. Juanes, Phys. Rev. E 84, 066312 (2011).

[24] T. Le Borgne, M. Dentz, P. Davy, D. Bolster, J. Carrera, J.-R. de Dreuzy, and O. Bour, Phys. Rev. E 84, 015301(R) (2011).

[25] J. J. Hidalgo, J. Fe, L. Cueto-Felgueroso, and R. Juanes, Phys. Rev. Lett. 109, 264503 (2012).

[26] M. A. Amooie, M. R. Soltanian, and J. Moortgat, Geophys. Res. Lett. 44, 3624 (2017).

[27] E. T. Hester, M. B. Cardenas, R. Haggerty, and S. V. Apte, Water Resour. Res. 53, 3565 (2017).

[28] D. R. Lester, A. Bandopadhyay, M. Dentz, and T. Le Borgne, Transp. Porous Media 130, 903 (2019).

[29] A. M. Tartakovsky, G. Redden, P. C. Lichtner, T. D. Scheibe, and P. Meakin, Water Resour. Res. 44, W06S04 (2008).

[30] D. Bolster, F. J. Valdés-Parada, T. Le Borgne, M. Dentz, and J. Carrera, J. Contam. Hydrol. 120-121, 198 (2011).

[31] M. Rolle, D. Hochstetler, G. Chiogna, P. K. Kitanidis, and P. Grathwohl, Transp. Porous Media 93, 347 (2012).
[32] P. de Anna, J. Jimenez-Martinez, H. Tabuteau, R. Turuban, T. Le Borgne, M. Derrien, and Y. Méheust, Environ. Sci. Technol. 48, 508 (2014).

[33] M. Muniruzzaman, C. M. Haberer, P. Grathwohl, and M. Rolle, Geochim. Cosmochim. Acta 141, 656 (2014).

[34] M. Icardi, G. Boccardo, D. L. Marchisio, T. Tosco, and R. Sethi, Phys. Rev. E 90, 013032 (2014).

[35] G. Chiogna, O. A. Cirpka, M. Rolle, and A. Bellin, Water Resour. Res. 51, 261 (2015).

[36] Y. Ye, G. Chiogna, O. A. Cirpka, P. Grathwohl, and M. Rolle, J. Contam. Hydrol. 172, 33 (2015).

[37] M. Boon, B. Bijeljic, B. Niu, and S. Krevor, Adv. Water Res. 96, 266 (2016).

[38] G. Chiogna, O. A. Cirpka, and P. A. Herrera, Transp. Porous Media 111, 591 (2016).

[39] E. Crevacore, T. Tosco, R. Sethi, G. Boccardo, and D. L. Marchisio, Phys. Rev. E 94, 053118 (2016).

[40] J. P. Bennett, C. P. Haslauer, and O. A. Cirpka, Water Resour. Res. 53, 2813 (2017).

[41] M. Rolle, R. Sprocati, M. Masi, B. Jin, and M. Muniruzzaman, Water Resour. Res. 54, 3176 (2018).

[42] R. Turuban, D. R. Lester, T. Le Borgne, and Y. Méheust, Phys. Rev. Lett. 120, 024501 (2018).

[43] Y. Ye, G. Chiogna, C. Lu, and M. Rolle, Transp. Porous Media 121, 315 (2018).

[44] M. Di Dato, F. P. J. de Barros, A. Fiori, and A. Bellin, Water Resour. Res. 54, 2095 (2019).

[45] M. Bakker and K. Hemker, Adv. Water Resour. 25, 747 (2002).

[46] M. Bakker and K. Hemker, Adv. Water Resour. 27, 1075 (2004).

[47] K. Hemker, E. van den Berg, and M. Bakker, Groundwater 42, 234 (2004).

[48] K. Hemker and M. Baker, Water Resour. Res. 42, W12419 (2006).

[49] F. Staufer, Adv. Water Resour. 30, 2194 (2007).

[50] G. Chiogna, M. Rolle, B. Alberto, and O. A. Cirpka, Adv. Water Resour. 73, 134 (2014).

[51] E. Huber and P. Huggenberger, Hydrol. Earth Syst. Sci. 20, 2035 (2016).

[52] J. Heyman, D. R. Lester, R. Turuban, Y. Méheust, and T. Le Borgne, Proc. Natl. Acad. Sci. USA 117, 13359 (2020).

[53] Y. Ye, G. Chiogna, O. A. Cirpka, P. Grathwohl, and M. Rolle, Phys. Rev. Lett. 115, 194502 (2015).

[54] Y. Ye, G. Chiogna, O. A. Cirpka, P. Grathwohl, and M. Rolle, Phys. Rev. E 94, 013113 (2016).

[55] G. Nichols, Sedimentology and Stratigraphy (Wiley-Blackwell, Chichester, UK, 2009).

[56] P. Jussel, F. Stauffer, and T. Dracos, Water Resour. Res. 30, 1803 (1994).

[57] A. D. Stroock, S. K. W. Dertinger, A. Ajdari, I. Mezić, H. A. Stone, and G. M. Whitesides, Science 295, 647 (2002).

[58] A. Hazen, State Board of Health Massachusetts Annual Report No. 24, 1892, p. 541.

[59] C. M. Haberer, M. Rolle, S. Liu, O. A. Cirpka, and P. Grathwohl, J. Contam. Hydrol. 122, 26 (2011).

[60] O. A. Cirpka, F. P. J. de Barros, G. Chiogna, M. Rolle, and W. Nowak, Water Resour. Res. 47, W06515 (2011).

[61] G. Chiogna, C. Eberhardt, P. Grathwohl, O. A. Cirpka, and M. Rolle, Environ. Sci. Technol. 44, 688 (2010).

[62] O. A. Cirpka and A. J. Valocchi, Adv. Water Resour. 30, 1668 (2007). 
[63] O. A. Cirpka, M. Rolle, G. Chiogna, F. P. J. de Barros, and W. Nowak, J. Contam. Hydrol. 138-139, 22 (2012).

[64] O. A. Cirpka, G. Chiogna, M. Rolle, and A. Bellin, Water Resour. Res. 51, 241 (2015).

[65] P. W. Atkins, Physical Chemistry (Oxford University Press, Oxford, UK, 1990).

[66] E. Worch, Vom Wasser 81, 289 (1993).

[67] D. H. Kelley and N. T. Ouellette, Nat. Phys. 7, 477 (2011).
[68] R. Lee, D. H. Kelley, K. N. Norfstrom, N. T. Ouellette, and W. Losert, New J. Phys. 15, 025036 (2013).

[69] M. L. Falk and J. S. Langer, Phys. Rev. E 57, 7192 (1998).

[70] G. Chiogna, D. L. Hochstetler, A. Bellin, P. K. Kitanidis, and M. Rolle, Geophys. Rev. Lett. 39, L20405 (2012).

[71] G. Chiogna and M. Rolle, Water Resour. Res. 53, 7488 (2017).

[72] O. A. Cirpka, A. Olsson, Q. Ju, M. A. Rahman, and P. Grathwohl, Groundwater 44, 212 (2006). 